University of Texas at El Paso

\title{
DigitalCommons@UTEP
}

Open Access Theses \& Dissertations

2017-01-01

\section{Analysis of gesture frequency and amplitude as a function of personality in virtual agents}

Alex Michael Rayon
University of Texas at El Paso, amrayon2@miners.utep.edu

Follow this and additional works at: https://digitalcommons.utep.edu/open_etd

Part of the Computer Sciences Commons

\section{Recommended Citation}

Rayon, Alex Michael, "Analysis of gesture frequency and amplitude as a function of personality in virtual agents" (2017). Open Access Theses \& Dissertations. 736.

https://digitalcommons.utep.edu/open_etd/736

This is brought to you for free and open access by DigitalCommons@UTEP. It has been accepted for inclusion in Open Access Theses \& Dissertations by an authorized administrator of DigitalCommons@UTEP. For more information, please contact lweber@utep.edu. 


\title{
ANALYSIS OF GESTURE FREQUENCY AND AMPLITUDE AS A FUNCTION OF PERSONALITY IN VIRTUAL AGENTS
}

\author{
ALEX MICHAEL RAYON
}

Master's Program in Computer Science

APPROVED:

David G. Novick, Ph.D., Chair

Nigel Ward, Ph.D.

Meagan Vaughan Kendall, Ph.D.

Charles Ambler, Ph.D.

Dean of the Graduate School 


\section{Copyright (C)}

by

Alex Michael Rayon

2017 


\title{
ANALYSIS OF GESTURE FREQUENCY AND AMPLITUDE AS A FUNCTION OF PERSONALITY IN VIRTUAL AGENTS
}

\author{
by \\ ALEX MICHAEL RAYON,
}

THESIS

Presented to the Faculty of the Graduate School of

The University of Texas at El Paso

in Partial Fulfillment

of the Requirements

for the Degree of

MASTER OF SCIENCE

Department of Computer Science

THE UNIVERSITY OF TEXAS AT EL PASO

May 2017 


\section{Acknowledgements}

Thank you to all my friends, mentors, and family that have supported me through this journey. When I had first started my college career, I never would have imagined that I would be working with virtual agents! Our lab has presented our agents across the world, and we have even won several awards. I am excited to be sharing what I have learned and I hope you all enjoy it.

Thank you to my mentors and committee members Dr. David Novick, Dr. Nigel Ward, and Dr. Meagan Kendall for you unending support and input. Each of you has contributed in many ways to me being where I am today. Special thanks to Dr. Novick for introducing me to the world of embodied conversational agents and all their capabilities. Also for inspiring me to pursue a Master's degree; you made every project that I worked on fun and satisfying. I will always cherish the values I have learned from you.

To my parents, thank you for all your patience and for instilling in me the drive to never give up. Thank you to my brother, Eric, who got me interested into the engineering field from an early age. I remember you bringing home projects, working on them with friends, and having a good time doing it. Thank you to my sister-in-law, Kate who motivated me to go for my Master's by getting her own first; looks like it's Eric's turn! To the rest of my family, thank you for understanding the amount of time I had to dedicate to my work, and loving me unconditionally. I hope I made you all proud!

Finally, thank you to my ISG family. Ivan, Diego, Mario, Adriana, Paola, Tim, Michelle, Saif, Aaron, Laura, Alfonso, Chelsey, Victoria, Brandon, Julie, Oliver, and everyone else over the years, it has been so much fun working with you. It was about 5 years ago we were a small team of five working on the Survival on Jungle Island, and now we are an award-winning lab with several projects under our belt. I wish the best to all of you! 


\begin{abstract}
Embodied conversational agents are changing the way humans interact with technology. Agents are expected to behave like people, both verbally and non-verbally. Researchers within the Human-Computer Interaction community have found many attributes of an agent's gesture contribute to an agent's perceived personality and believability.

Gesture amplitude and frequency are two of the attributes that contribute to the naturalness of a gesture. Currently, no previous studies have defined an empirical baseline for generating natural gestures for these two attributes. I seek to discover whether gesture amplitude and frequency affect how users perceive an agent's naturalness, and which gestures would be preferred for an agent based on its personality.

In particular, I seek to quantify gesture amplitude and to compare it to a previous study on the perception of an agent's naturalness of its gestures. In this thesis, non-verbal gesturing consists of movement of the arms and their location in space. I evaluate whether agents should use specific gestures more frequently than others depending on the personality type they have been designed with. My study is organized into three experiments and evaluations. My results show some indication that introverts and extraverts judge the agent's naturalness similarly. The larger the amplitude the agent used, the more natural its gestures were perceived. The frequency of gestures between extraverts and introverts seem to show hardly any difference, even in terms of types of gesture used.
\end{abstract}




\section{Table of Contents}

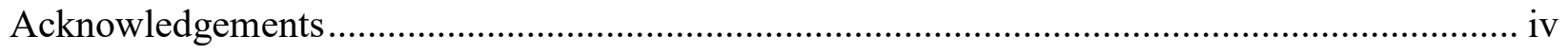

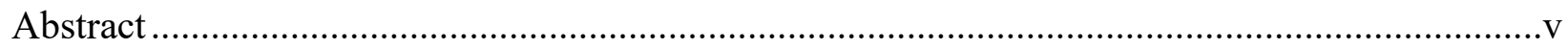

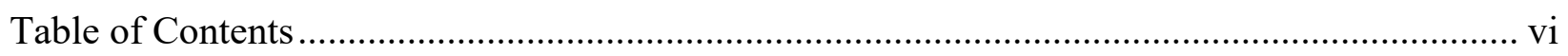

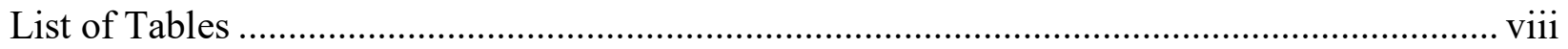

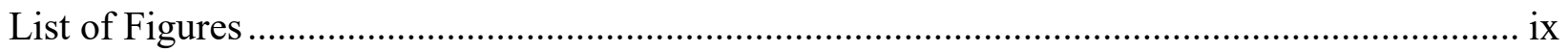

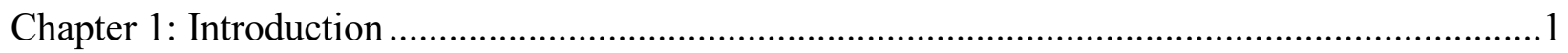

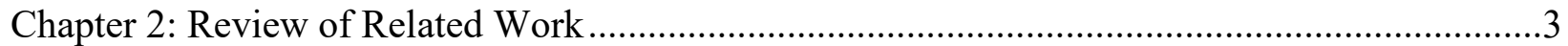

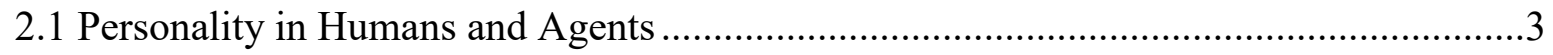

2.2 Gesture in Human-Agent Conversation................................................................5

2.3 Gesture Amplitude and Frequency ..................................................................... 7

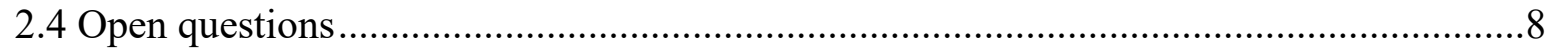

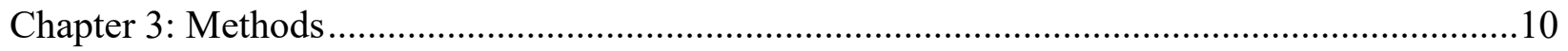

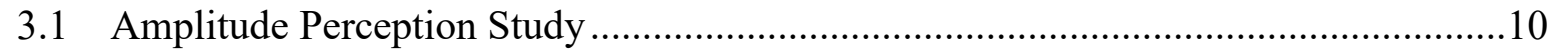

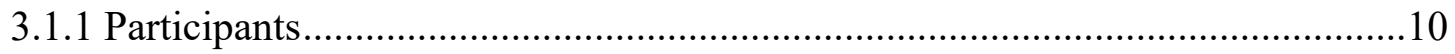

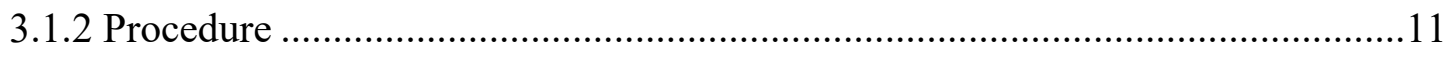

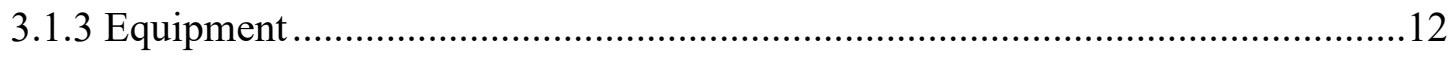

3.2 Amplitude Measurement Study .................................................................... 13

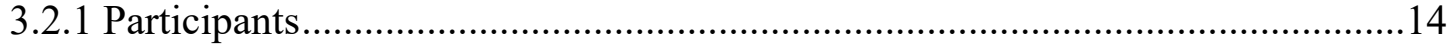

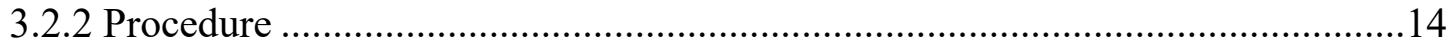

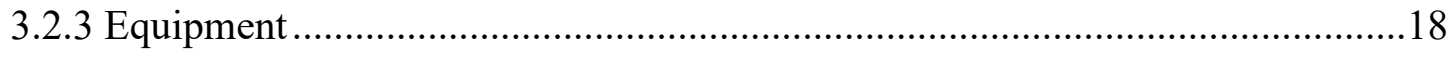

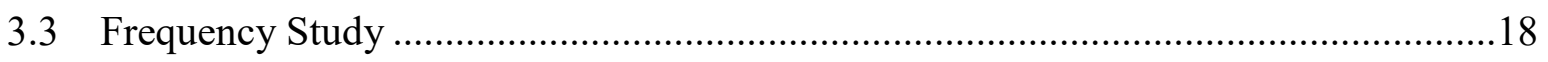

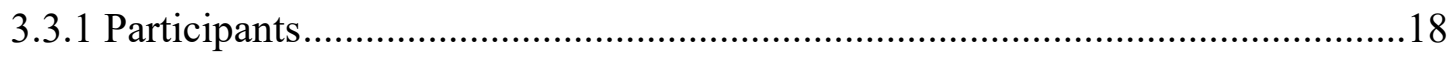

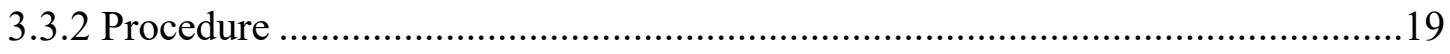

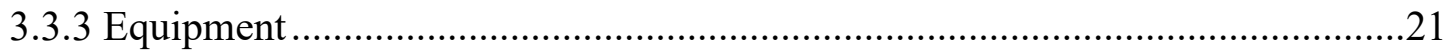

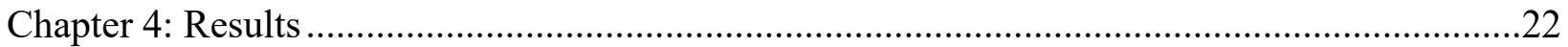

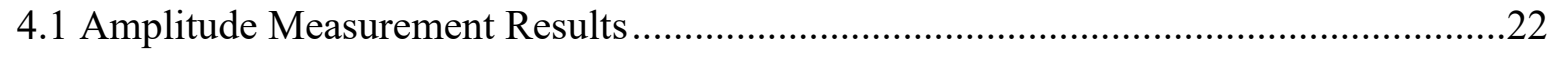

4.2 Amplitude Perception Results..............................................................................24

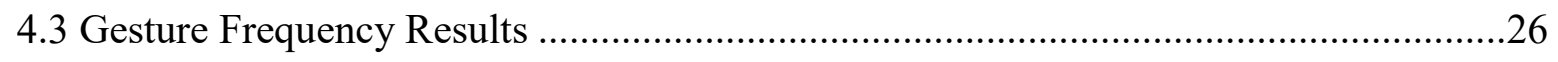


Chapter 5: Discussion

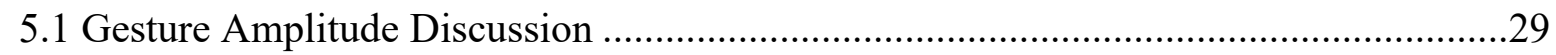

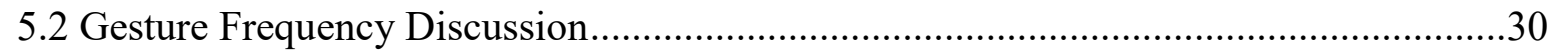

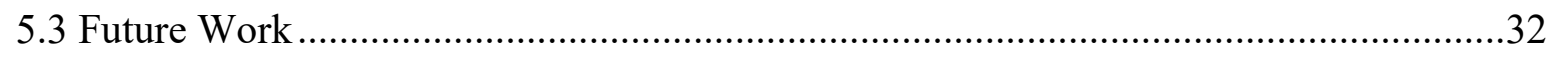

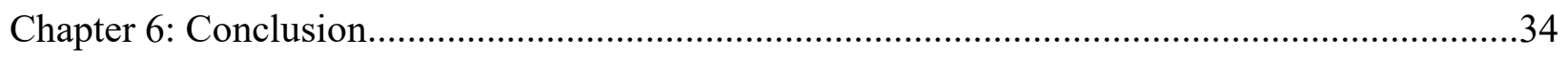

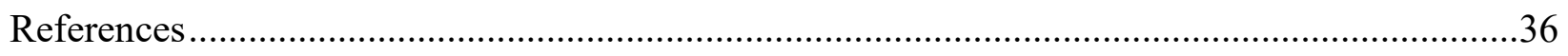

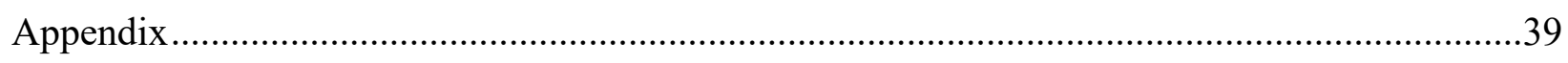

Vita 41 


\section{List of Tables}

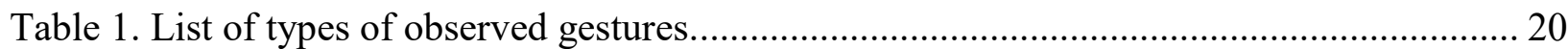

Table 2. Annotations of three beat gestures......................................................................... 20

Table 3. The amplitude vector for each arm was added and the gestures were sorted to create an even distribution across the small, medium, and large categories........................................ 23

Table 4. Median for each amplitude category and its relation to the naturalness perception study

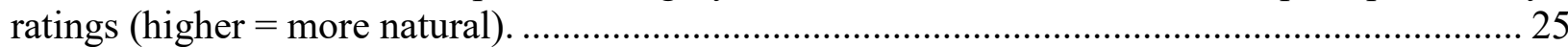

Table 5. The minimum and maximum gestures per minute for each personality type, along with

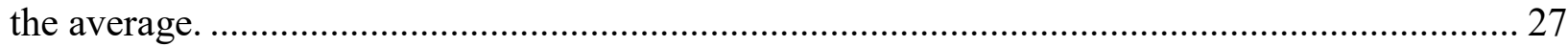




\section{List of Figures}

Figure 1. Gesture from each category from left to right: A, B ,C, I, and E. A has the smallest amplitude and $\mathrm{E} / \mathrm{C}$ has the largest.

Figure 2. An initial version of a way to measure the agent's amplitude. In this example measuring to the fingertips would give 9,9 for the right hand and 9,8 for the left. 15

Figure 3. The same point in time for measuring the apex of the right hand in Gesture A4.

Displacement is measured from where the hand would be in the neutral position to the apex of the gesture. 17

Figure 4. Two participants conversing. The introverted subject (left) uses the same Arms Out gesture as the extravert (right) but his arms are rotated inwards closer to his body. 19

Figure 5. The annotators were in the same room but were only allowed to converse about a gesture after everyone had rated it. 21

Figure 6. Mean amplitudes (in inches) of the gestures to be used in the perception study. Each gesture amplitude was calculated as the maximum of the the right-arm and left-arm vectors..... 23 Figure 7. The rating of amplitude overall for each set of animations (A to $\mathrm{C}$ increasing in amplitude, I and E previously used animations). A score of 4 represents the amplitude judged most natural.... 24 Figure 8. Perceived amplitude vs. actual amplitude. A score of 4 represents the amplitude judged most natural. The actual amplitude represents the maximum of the the right-arm and left-arm vectors. 25

Figure 9. Median for each amplitude category and its relation to the naturalness perception study

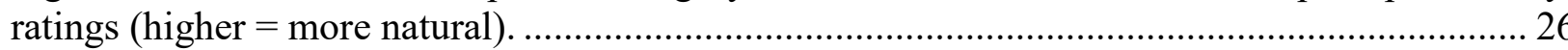

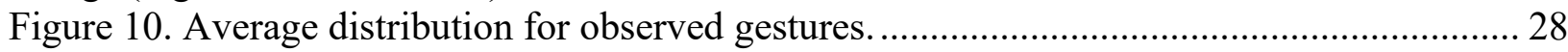




\section{Chapter 1: Introduction}

The future with how we interact with our virtual space is changing rapidly. The field of human computer interaction is expanding with systems and interfaces that have an embodied presence. Virtual agents, also known as embodied conversational agents (ECAs), have linguistic capabilities and, in many cases, can perform gestures and facial expressions using a virtual body (Cassell, 2000). Research suggests that users may prefer ECAs as an interface to a virtual system because they feel more comfortable speaking to a computer if what they are interacting with has a visible presence (Novick and Gris, 2014). Creating a humanlike ECA is difficult because it requires knowledge of complex social features like gestures, personality, and the role they play in interaction. Indeed, the more humanlike an ECA acts the easier it is to develop rapport with it (Clausen-Bruun et al., 2013). Rapport is the sense of connection and mutual understanding between two people that is attained through interaction and conversation. People develop rapport with one another at different rates, and is difficult to model because it is subjective. Both verbal and non-verbal actions can contribute to rapport building behaviors.

In this thesis I focus on the development of non-verbal behavior and its effect on user perception of human likeness and personality. Specifically, I investigate the naturalness of nonverbal gestures that involve arm and hand movement. Gesturing contains many messages that we subconsciously identify and make judgments from (McNeill, 1985). One of these is personality of the agent and the naturalness of its gestures (Hu et al., 2015; Neff et al., 2008; Neff et al., 2010). Personality is comprised of many dimensions, but for this thesis I will focus on the dimension of introversion/extraversion. People can show extraverted and introverted traits, but most tend to lean towards one side. Extraverts can be defined as talkative, and they thrive off the energy from social interactions. Introverts tend to be more reserved, and reflective on themselves (Jung, 1971). Although past studies have viewed how these personality traits contribute to gesture and interaction in agents, some of the least investigated are their effects on gesture amplitude and 
frequency (Clausen-Bruun et al., 2013; Kipp et al., 2006; Gratch 2007). A survey of the literature disclosed no research detailing amplitude or frequency in empirical terms.

The goal of this research is to define an empirical baseline for the naturalness of gesture amplitude and frequency so that interactions with ECAs can elicit humanlike behavior that adjusts to the personality of the user. Gesture amplitude is the size of a person's movements, and for this thesis focuses on the movement of the arms. Gesture frequency is how often a person gestures as well as the type of gestures they use. In this thesis, frequency is defined by gestures per minute. I believe that precise amplitudes of gestural stimuli can determine whether users of differing personality types view gesture amplitude differently. It is quite possible that an introverted person viewing an extraverted agent could view those extraverted gestures as unnatural. My hypothesis is that both personality types will view the largest gestures as natural in amplitude. If there is no difference in perception, then ECA creators can design an agent to use the gesture amplitude size that is viewed as natural for both user personality types. Essentially a baseline of natural gesture amplitude could be made that defines size ranges for gestures that appear most natural. As for gesture frequency, I want to determine whether personality type factors into specific gesture use, gesture type, and rate of gestures people use. Knowing what gestures introverts and extraverts use more, and how often they gesture could be utilized in agents to help in "giving off" the intended personality for the agent.

In the following section I review the concepts of ECAs, their design, and the current state of gesture research. I explain the methodology and experimental set-up for each study conducted along with the results. Finally, I will discuss new aspects of gesture amplitude and frequency, and conclude with possible future work and insights. 


\section{Chapter 2: Review of Related Work}

This thesis relates to research from several different fields of study. It is essential to understand each concept and how it contributes to the overall idea: gesture's role in the perceived personality and naturalness of an agent. This review will be split into four sections: the role of personality in humans and ECAs, role of gesture in conversation, the importance of gesture amplitude and frequency, and the questions that arise from previous studies.

\subsection{Personality in Humans ANd Agents}

An ECA is a form of human-computer interaction that involves an intelligent virtual character that can communicate by using speech, facial expressions, and gestures (Cassell, 2000). ECAs can vary graphically in appearance depending on the desired virtual environment the ECA lives in and the role assigned to them. A help-desk ECA may only have its upper body visible, while a museum's tour-guide ECA may need a full body to convey more lifelike gestures and behavior (Swartout, 2010). ECAs that appear more human-like are easier for humans to interact with and develop rapport with(Clausen-Bruun et al., 2013). Their combination of gesture, speech, and facial expressions factor not only into believability and rapport but also into the perceived personality of the agent (Neff et al., 2008). ECAs are designed to be used in conversational settings. They should be able to handle the discourse within a conversation and respond in humanlike ways to input (Bickmore \& Cassell, 2001). Based on these findings, I conclude that ECAs that do not behave in the same humanlike manner that is expected from them may ultimately lose respect and rapport from the user.

To meet the high expectations of users, several features must be considered when designing an ECA, depending on its application. Extraversion, agreeableness, and other Big Five personality traits are important to an ECA's design (Cerekovic et al., 2014). Extraversion is being talkative, outgoing, and enjoying social interactions (Jung, 1971). Users show higher levels of rapport when interacting with extraverted agents even if the users themselves are not extraverted (Brixey, 2015). 
This may be because extraverts can be perceived as seeking the company of others and exhibit positive emotions in their behavior (Ivanov, et al, 2011; McCrae \& Costa Jr., 1997). Introverts are characterized by the opposite: they like keeping to themselves, making decisions by reflecting on internal conversations, and avoiding social interactions. These personality traits can be expressed not just through an agent's speech but also non-verbal behavior (Neff et al., 2008, 2010).

Personality is an important aspect of what defines a person, and, in turn, an ECA. In humans, personality plays a role in choosing which relationships and friendships to pursue (Eysenck, 1985; Botwin et al., 1997). Personality can be defined as "the combination of characteristics or qualities that form an individual's distinctive character." What people think, and how they behave (their character) can be gathered from their personality. Although speech is a factor in perceiving a person's personality, non-verbal cues and gestures provide an equally important facet (Argyle, 1975). As humans, we are constantly evaluating the people around us, whether we are aware of it or not. In a conversation, this includes the personality of a person. Through observing gesture and dialog, we make judgments of a person's character.

ECAs should "give off" the extraverted personality that resonates with most people so that engagement is maintained (Brixey, 2015). However, extraversion is not the only trait that contributes to personality. The Big Five Factor model includes four other defining traits for determining the personality of a person: agreeableness, openness to experience, conscientiousness, and neuroticism (Digman, 1990). Agreeableness is characterized by being considerate of others, and willing to compromise. Openness to experience refers to wanting to try new things and being intellectually curious about the world around one. Conscientiousness is characterized by being goal-oriented, planning ahead, and desiring organization. Neuroticism is the level of emotional instability and the degree to which negative emotions control a person's actions. Within this model personality can be categorized as a person showing a "high" or "low" affinity for each trait (McCrae \& Costa Jr., 1997). This thesis is concerned only with observing the level of extraversion, which has been shown to be representative of people who enjoy socialization and give the feeling 
of warmth/comfortableness (Digman, 1990; McCrae \& Costa, 1997). Extraversion may play a large role when determining who we decide to trust and with whom we develop rapport.

While gestures do not have defined parameters for expressing intended personality traits, studies of human-human interaction can be used as a basis for identifying personality. Indeed, in human-human conversation, most of the message being communicated was found to consist of nonverbal behavior (Mehrabian \& Ferris, 1967). This study had the investigators observing speech and non-verbal association as a test. Speech and nonverbals were classified as three different degrees (positive, neutral, and negative), and different combinations were used to see which had more weight. Nonverbals were found to have more weight (55\%) than the speech component (38\% for voice \& $7 \%$ for words), but both together were the best way to communicate a message.

There are several different types of nonverbal gestures, each with a different level of meaning behind the message (McNeil, 1985). This thesis will focus on three types: iconic, deictic, and beat. The first type comprises iconic gestures, which relate closely to speech. They help illustrate what is being said and are useful when synchronized with speech. An example of an iconic gesture would be using hands to indicate an object's size. The second type comprises functional/deictic gestures, which imply instruction to a person, usually by pointing to a space. An example of this would be pointing to a location and telling someone to move to that spot. The third type comprises beat gestures, which consist of rhythmically moving a body part; these are usually created spontaneously during speech. Speakers also use beat gestures to emphasize words by pausing the rhythm of speech. For example, person presenting a talk may tend to move both hands in small circular motions and slow down these motions when saying key words.

\subsection{Gesture in Human-Agent Conversation}

With their importance in human-human conversation, gestures likewise play an important role in human-agent interaction (Hostetter, 2012; Kendon, 1980; Kendon, 1986). ECAs that are able to understand a user's gesture, to respond with their own gestures, and to synchronize this with their speech represent an ideal in this regard. The problem is choosing the correct gesture and 
expressing it in a manner consonant with the user's personality, the dialog, and the user's beliefs about the agent's capabilities. Once performed, gestures in turn could contribute to the user's perception of the agent's personality.

First impressions are an important part of the conversational process, which can shape how a user interprets an ECA's persona. Within the first 12.5 seconds of interaction with an ECA, users will make judgments about friendliness, likeness, and extraversion (Cafaro et al., 2012). This conclusion was reached via an experiment in which the agent would approach the users with varying levels of gaze, smile, and proximity to the user; ECAs that smiled were seen as more friendly and likable even if they approached the user before a conversation. Thus an ECA that intends to be friendly should start its a conversation with a smile. This result is consistent with findings in social psychology that smiling when starting a conversation indicates extraversion (Niedenthal, 2010).

For ECAs, because they are typically designed to be as humanlike as possible, the idea of utilizing gestures used by humans seems appropriate. Previous studies have created animations based on recorded human gestures by either using motion capture or copying movement from video (Bergmann et al., 2010; Clausen-Bruun, 2013; Neff et al., 2008). From these studies, the gestures that were rated by users were mostly seen as natural and humanlike. Natural qualities are important for the agent because if the agent is perceived as unnatural, then it will likely be more difficult to build human-agent rapport (Gratch, et al., 2007). However, what is considered a "natural" gesture to capture is problematic because the process of capturing gesture from humanhuman interaction may not be natural in itself. Even if gestures are captured in a non-intrusive way, the range of amplitude for naturalness that could alter the personality perceived by the user may not be known. And the amplitude of gestures that seem appropriate in human-human interaction may not necessarily seem appropriate in human-agent interaction. Moreover, the type of gesture and the personality of the person who was used for capturing the gestures may also affect the agent's perceived personality. If the developers of an ECA wish to have the agent convey 
a particular personality, then the capture, processing, and generation of gestures will have to take this design goal into account.

\subsection{GESTURe AMPLitUde AND FREQUENCY}

A study (Neff et al., 2008) suggested that different levels of gesture rate and amplitude have been tested to see if they affect an agent's perceived level of extraversion. This study's investigators developed a gesture rubric that included several nonverbal motions that comprise the gesture. The rubric included gesture amplitude, arm swivel, stroke, duration of the gesture, and much more. Their results indicated that each of the attributes contributed to increased extraversion perceived by the user. However, the gestures' amplitude was described only in general terms with respect to differences between extraversion ("broad") and introversion ("narrow"). Another study (Clausen-Bruun et al., 2013) focused on the amplitude feature from the rubric. The amplitude of gestures was measured with visually different agents to see which one users connected more with and which one provided more information uptake. Information uptake was defined as what information told by the agent the user retained after their interaction. Users listened to a story told by two agents, a cartoon alien agent and a realistic human agent. Both agents used the same motion-capture movements for the gestures, but the human was perceived as having more natural gestures and gestures that better facilitated information uptake. Participants rated the alien as more distracting because of its appearance, and that it was harder to establish mental representations of the story it was telling. Regardless, users remembered information more in the large-amplitude extraverted condition of the videos than the small-amplitude introverted condition regardless of character. This means that gesture amplitude does impact what users interacting with an agent will remember from a conversation. Combined with appearance of the agent, amplitude also affects how natural the agent appears to be. I note, though, that this study did not define a baseline for the amplitude, which led to having a high baseline for the normal gestures. Their use of motion capture for their agents' gestures involved using an actor in a full body suit being told what to do. This lead to such a high amplitude level for their normal amplitude condition. For future systems, if a 
baseline amplitude for normal gestures were known, an agent could adjust gesture amplitude to convey personality. Accordingly, in the series of studies reported here, I work toward defining just such a baseline.

Another study (Hu et al., 2015) found that users notice if an agent is extraverted, based on the amplitude of its gesture. This study used a storytelling scenario between two agents to see if users perceived the personality of each agent. One agent would gesture with large amplitude while the other used gestures that were the same but smaller in size. Users noticed the difference between the two agents and correctly perceived that the larger-amplitude agent was the extraverted agent. However, though amplitude does contribute users' perceptions of agents' personality, there may be a threshold for the amplitude. That is a gesture that is too big or small could be seen as unnatural. Accordingly, in experiments reported here, I seek to calibrate the size and the perception of size of gestures across extraverted/introverted agents and across extraverted/introverted subjects.

Personality appears to affect conversants' gesture rate. In particular, extraversion correlates positively with the production of "representational" (i.e., non-beat, iconic and deictic) gestures (Hostetter, 2012). The study grouped participants in pairs to play a word game where one participant had to guess a word by listening to the other describe the word through speech. The experimenters observed their gesture frequency and how it related to their self-reported levels of extraversion. The believe that the reason extraverts perform more representational gestures is because they either have more energy, gesturing has become habitual, or a combination of both. This research leads to open questions of whether these findings translate to more realistic conversational settings and whether a similar pattern obtains for beat gestures.

\subsection{OPEN QUESTIONS}

The research reviewed above suggests that agents who perform gestures with high amplitude and frequency do appear extraverted to users. A problem with many of these studies, though, is the lack of a scale to define the amplitude of the gestures. The studies omit clear full-body measurements of the agent in relation to its gestures. The gestures themselves are given a range 
between extraverted and introverted on a 3D plane, but there is no measurement of the initial and apex of a gesture. There could be a limit: if a gesture is too large then it may seem unnatural. The same logic applies to gestures that are too small.

Accordingly, in this thesis I seek to answer two questions:

1. Do people perceive an agent's gesture as more or less natural as a function of (a) the person's personality trait of extraversion vs. introversion and (b) the amplitude of the agent's gestures?

2. Do the perceived sizes of the gestures correspond to their actual sizes?

The answers to both hypothesis would lead to the creation of a baseline for natural sized gestures that would differ based on personality type of the user. An extraverted user may perceive the naturalness of a given gesture differently from an introvert who may see the same gesture as unnatural. Likewise, the opposite may be true in which both personality types share the same perception of amplitude which would mean the creation of a singular naturalness baseline.

Also the type of gestures used by extraverts may differ from those used by introverts. If that is the case, then agents designed to be extraverted should use those specific gestures more frequently over the more introverted ones. Introverts tend to perform gestures that are closer to their bodies (Argyle 1975), but in most studies done with ECAs an introverted gesture is the same gesture used by the extrovert, just smaller. My review of the literature has disclosed no study that has looked for diversity of gestures across these personality traits. So it is possible that users may classify an agent as introverted not only on the size of its gestures but on the different types of gesture it uses.

This leads to the third question:

3. Which personality type gestures more frequently, and are there any gestures that are more frequently used by each one?

Even if a baseline of gestures is created, I still want to know whether personality types vary in frequency, and use specific gestures exclusively or more frequently than one another. The answer to this hypothesis would allow ECA designers to adjust gesture frequency to suit the personality type of the user. If a range of frequencies for each personality are empirical, then designers can follow them as a guideline, which is something that does not exist as of this writing. 


\section{Chapter 3: Methods}

This thesis tests several aspects of gesture captured from natural conversation. I conducted three studies using the same source for the gestures but that focused on different hypotheses: a study of gesture amplitude perception, a study of gesture amplitude measurement, and a study of gesture frequency. Because each study was its own self-contained experiment, I will describe each one's methods, including participants, procedure and equipment, in its own section.

\subsection{Amplitude Perception Study}

This study will seek to answer the first question, which is do people perceive an agent's gesture as more or less natural as a function of (a) the person's personality trait of extraversion vs. introversion and (b) the amplitude of the agent's gestures?

If users perceive the naturalness and amplitude similar between personality types, then any gestures created in the future would need to be modeled after the most natural ones.

\subsubsection{Participants}

Participants were recruited by word of mouth and by asking professors of the Computer Science Department of the University of Texas at El Paso to recruit students from classes. An extra credit incentive was offered to students in these classes. Other participants received no incentive. There were no specific requirements needed to participate in the experiment. Participants only needed to be able to watch the agent projected against the wall and to write down their judgments for each gesture on a sheet of paper.

In total there were 60 participants, 15 female and 45 male. Participants took a brief introversion/extraversion-scale survey. The survey consisted of a standard Myers-Briggs personality assessment that used a word-pair approach (similarminds.com). This survey measured four aspects of personality: extraversion/introversion, intuitive/sensing, feeling/thinking, and perceiving/judging. The survey labeled each dimension with a percentage that represented how likely that participant belonged to a given trait. In the case where a subject fell evenly into both dimensions ( $50 \%$ introvert and extravert), they were placed in the personality group that needed 
more people. I wanted to make each personality group have similar total number of subjects. From the 60 participants, 30 were classified as extraverts and the others as introverts.

\subsubsection{Procedure}

All participants were told that the experiment would take about 20 to 30 minutes to complete. They were also informed about the risks of completing the experiment and given a consent form to sign. After signing, the participants took the personality survey and their results were recorded. Participants were seated within UTEP's Immersion Lab and given a paper that provided an example of a 7-point Likert scale and 30 empty spaces to fill in. Each empty space corresponds to a gesture and each subject wrote their numerical rating within this blank space. They were told that they were to judge the size of the agent's gesture based on the scale in which 1 represents unnaturally small gesture size and 7 represents unnaturally large gesture size. Each personality group rated every single gesture from each category for a total of 30 rated gestures per subject. The agent did not perform any hand or facial animations, so participants were asked not to judge based on those parts. The agent performed lip-sync animation with its mouth but did not have audible speech.

Participants were brought into the experiment space in groups, depending on the time they signed up for. In total, there were 14 groups ranging from 1 to 11 participants per group. Once all subjects were ready, a demonstration of five gestures was shown so that participants could get a feel for the agent's movement and could ask any questions. Once the demonstration was completed, each gesture was played on a continuous loop until all participants were ready to move on to the next gesture. The experimenter controlled when the next gesture would be played. Participants were not allowed to converse with one another while viewing the gestures. They were also each seated within their own chair and told not to copy off each other. Once a group was done, their data were recorded and they were escorted back to the waiting area. The gestures were shown in a non-repeating random order for each group of participants. 


\subsubsection{Equipment}

The agent and its environment were developed using the Unity 3D game engine software. The ECA was built using the software architecture developed by the Advanced aGent ENgagement Team research group at the University of Texas at El Paso. This agent has capabilities similar to those of the agent used for the Survival on Jungle Island game (Novick, 2015). As a small clarification, this agent has been used in previous experiments and demos in the lab and viewed by both genders with no qualms about her appearance. For the perception study, the agent's ability to recognize speech and gesture was removed. The agent needed only to perform the gestures for participants to rate it. The agent did not speak, because this could have affected how the participants perceived its personality. The agent was projected onto a wall so that all participants could view her gesture. The sequencing of the agent's gestures was controlled manually from the experimenter's station so that all participants could take their time rating each gesture. A gesture was played through its full animation and continuously looped until all participants were ready to move on. This means that although some groups saw the gesture looping more than others, all groups viewed the entire length of a gesture which is the focus of the rating scale.

The gestures were separated into five categories. Three of the categories $(A, B, C)$ were gestures captured from human-human conversation that increased in amplitude. These gestures were captured using two Microsoft Kinects to record the movements of each participant. They were separated into A, B, and C categories by what the experimenters thought were gestures of increasing amplitude. At the time of this experiment, I was not focused on figuring out how to measure gesture amplitude and was instead following knowledge from past research of what makes a small/large gesture to make the categories (Neff, 2008). The other two categories (I, E) acted as a control group. The control-group gestures were animated from a previous experiment, with the E animations acting as modified versions of the I gestures but with a larger amplitude. In total there were 30 animations, six from each category. An example of a gesture from each category can be seen in Figure 1. The "A" gestures were small movements usually made close to the sides

of the agents body in most cases. "B" gestures were further from her sides and more forward. "C" 
gestures were expansive with both arms with the agent even rotating her body a bit in some cases. The "I" and "E" gestures had her moving her arms towards and away from her body with the "E" versions being amplified versions of the "I".

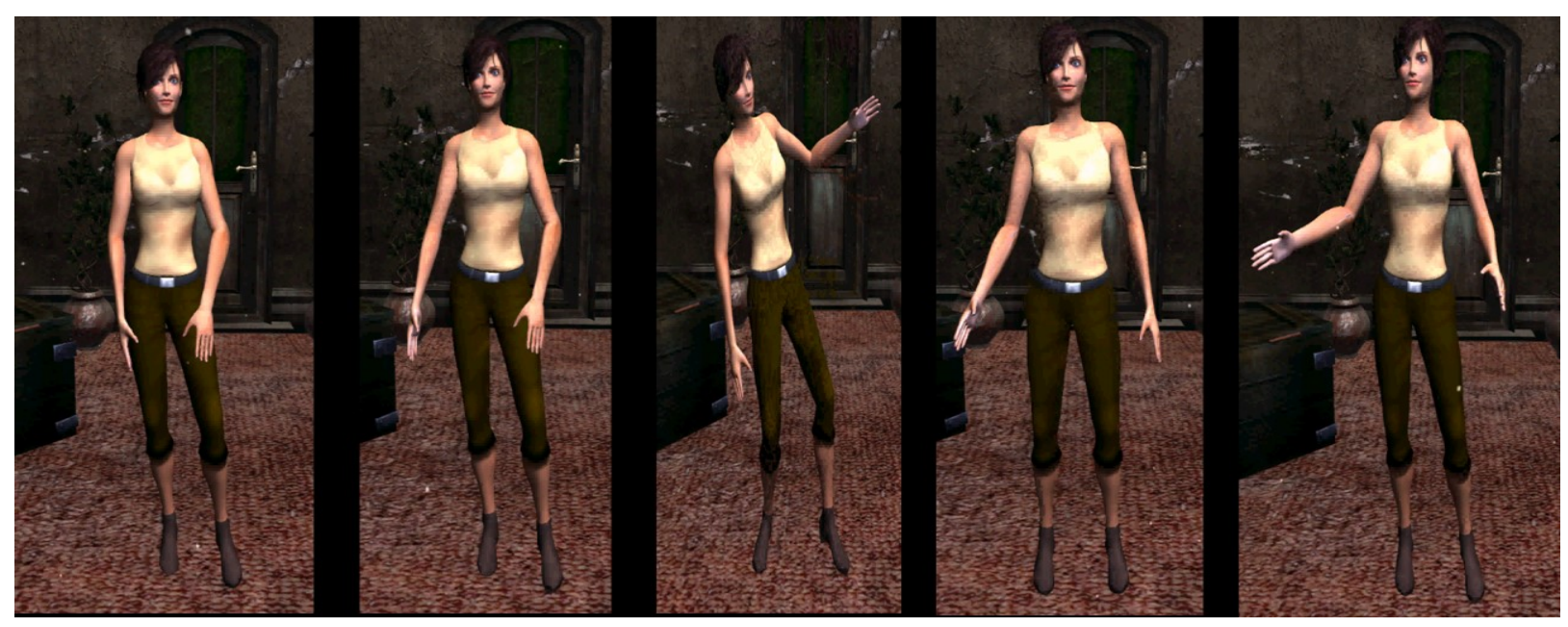

Figure 1. Gesture from each category from left to right: A, B ,C, I, and E. A has the smallest amplitude and $\mathrm{E} / \mathrm{C}$ has the largest.

As for the lab space, participants were seated across from the agent, which was projected onto the wall to appear life-size. Participants were close to one another in some cases but were asked to take their time answering and not to talk with one another.

\subsection{Amplitude Measurement Study}

Previous studies have reported how they altered gesture amplitude to create more extraverted gestures. The problem is that did not provide the details on how they created a baseline to determine what is and is not an extraverted gesture. The purpose of the second experiment was to define a baseline for the personality trait of extraversion based on the gestures used by the agent in Section 3.1. I also wanted to find the apex of amplitude for the gestures to see if they matched results from the amplitude perception experiment. The problem with creating this baseline was determining the proper way to measure amplitude of a gesture. 


\subsubsection{Participants}

This experiment did not require using participants from outside the lab space. Instead two annotators were given the task of measuring each of the agent's gestures. The annotators were research assistants from the Interactive Systems Group lab where each of the studies in this thesis took place. Two were chosen so that one could measure while the other wrote down the measurements.

\subsubsection{Procedure}

The annotators had to devise an unbiased way of measuring the agent's amplitude. A single animation can consist of multiple beat gestures before the intended gesture is performed by the agent because of the nature of the live capture. Keeping just the intended gesture may seem like the logical idea, but this lead to animation errors. For this study, the annotated gestures are from a full-body representation of the ECA, but annotation focused on the agent's arms. The agent had no finger or wrist movement in her gestures due to limit of recording live gesture with the Kinect. It is also important to note that the annotators had to agree on the gesture that appeared the largest during an animation to record the apex.

Figure 2 shows the first iteration of the measurement approach, which was a grid overlaid over different screenshots of the lowest and highest amplitude point of a gesture. This grid was designed so that the further out the agent's hands were, the larger her gesture was recorded. The size of the gesture was measured by looking at the coordinates where her hands were. The start and end positions of the perceived largest gesture were captured and measured. 


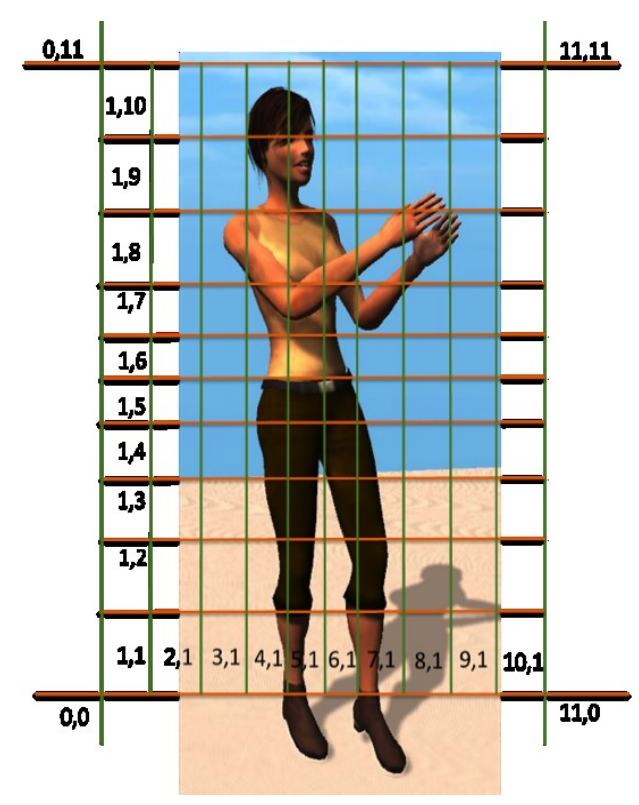

Figure 2. An initial version of a way to measure the agent's amplitude. In this example measuring to the fingertips would give 9,9 for the right hand and 9,8 for the left.

The problem with measuring gestures this way was that only the $\mathrm{x}$ and $\mathrm{y}$ axis could be analyzed. An origin was created by looking at the quadrants that the fingertips started at before the gesture began. There was no indication on the measurement for the z-axis of the gesture, which also contributes to the amplitude. Even if screenshots were taken from the side of the agent, this grid would not be a good indicator of the z-axis distance. Determining exactly what coordinates the fingertips were in was difficult as well. To eliminate possible error when determining the size of a gesture, the extremal spaces in the grid have a larger amount of space to ensure that the extremely large gestures are measured as such. I tried several iterations of the grid based measuring system, but each contained different faults. Another problem concerned using the hands as the measuring base for the starting point and apex point of a gesture. Gestures that would normally be classified as introverted (hands and arms inwards towards the chest) with a small amplitude would be measured as a large amplitude because they crossed over the agent's body. Figure 2 is a prime example, where the agent's right hand is measured as having a large amplitude on the $\mathrm{x}$-axis because it is crossing over her body. 
The solution to these problems was perhaps the most obvious: measure each gesture as though the agent was in the experiment space and determine a neutral position for the starting point so that cross-over measurement does not occur. If the displacement could be measured for each axis in 3D space, then the annotator would have an accurate value for the amplitude of a gesture. Accordingly, the agent was projected at the same size and on the very same wall as it had been projected in the perception experiment. That is, the agent was exactly the same size, and its gestures were exactly the same amplitude, as in the first study. For these measurements to be accurate, the starting point of each gesture (which is variant depending on said gesture) would need to have a shared neutral starting position. This became the neutral "hands by the side" position in which the agent starts before each animation. In this neutral position, her arms are at her side waiting for a gesture to be played. This pose is similar to anatomical position except with the palms of the hands facing inward.

The new measurement process consisted of the annotators pausing the agent and measuring the distance of her hands from the neutral position to the maximum amplitude of her gesture. Each measurement was made in inches using yardsticks placed against the projected wall. The horizontal and vertical distance in inches for her hand during maximum amplitude represented the $\mathrm{X}$ and $\mathrm{Y}$ axes. Rotating her model in Unity provided the annotators with a way to measure the $\mathrm{Z}$ axis. These measurements were done for both of her arms because most of her gestures were conveyed using both of them. Figure 3 shows an example of a measurement for the agent's right hand. A digital version of the measuring process was also attempted instead of measuring her from the projected wall. Within Unity there are ways to calculate the location of objects like characters and environment pieces in the space, but a reliable way to pinpoint the fingertips and origin point could not be established. 

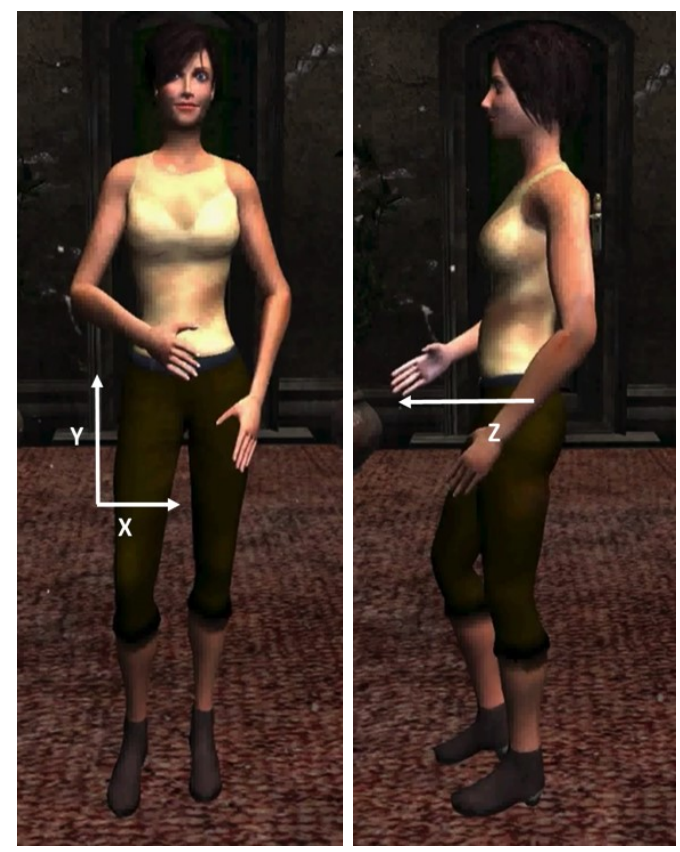

Figure 3. The same point in time for measuring the apex of the right hand in Gesture A4. Displacement is measured from where the hand would be in the neutral position to the apex of the gesture.

If a gesture had her crossing her hands over her body, the respective axis was marked as negative in displacement, but absolute value was used when calculating results. I defined negative displacement relative to the axis being measured from the starting position. If the agent's arms crossed over her body from left to right (or vice-versa for the other arm) then that was marked as negative displacement. Similarly, negative vertical displacement was when the gesture went below the starting position, and negative $\mathrm{Z}$ axis was when the gesture ended up behind the agent. After conducting the measurements, I calculated the total distance traveled by the hand using the distance formula: $\operatorname{sqrt}\left((\mathrm{x} 2-\mathrm{x} 1)^{2}+(\mathrm{y} 2-\mathrm{y} 1)^{2}+(\mathrm{z} 2-\mathrm{z} 1)^{2}\right)$. This calculation was performed for both the left and right hands for each gesture. The result is a vector for each arm that can be used to categorize the actual size of a gesture. Once all the gestures are categorized by vector size, a baseline of gesture size in relation to naturalness can be made using the results from 3.1. 


\subsubsection{Equipment}

The agent used for this study was the same one as in the perception study. The agent's gestures were recorded from the same environment in Unity. Yardsticks were used to measure the displacement of the agent's gestures.

\subsection{FREQUENCY STUdY}

The purpose of the third study was to discover if people within natural conversation differed in their use of gesture and gesture rate (frequency) based on their personality trait of extraversion. If extraverts used a specific gesturing pattern than introverts, then extraverted agents would need to be designed in a similar fashion. This study into frequency is exploratory, which suggested three hypotheses:

1. Extraverts will gesture more frequently than introverts.

2. There will be a significant difference between the frequency of specific gestures used between introverted and extraverted subjects.

3. Extraverts will have more variance in gesture types than introverts.

\subsubsection{Participants}

A previous experiment (Gris, et al. 2015) had recorded video of one-on-one conversations between people to capture gesture animations for our agent. These videos are the same ones used in 3.1 to create the $\mathrm{A}, \mathrm{B}$, and $\mathrm{C}$ gesture categories. The subjects were asked to complete an instrument indicating their extraversion/introversion trait. The subjects were isolated within a room so that they would gesture as naturally as possible while talking to one another. The pairs of subjects were grouped based on the Big Five personality trait of extraversion vs. introversion. They were grouped into introvert-introvert, extravert-extravert, and introvertextravert pairs. Subjects were tested for their personality type by using the Similar Minds word pair approach (Jung Word Pair Test). This survey provides an estimate of personality type by using percentages across four dimensions: extroversion/introversion, intuitive/sensing, feeling/thinking, and perceiving/judging. An example could be a participant being $70 \%$ extravert, 
and $30 \%$ introvert. In total, my colleagues and I recorded ten conversations/pairs, but only seven of these could be used for this study due to recording errors. Thirteen videos, each representing an individual in the conversation, were used for the analysis: seven extraverts and six introverts. Two participants can be seen in the experiment space in Figure 4.
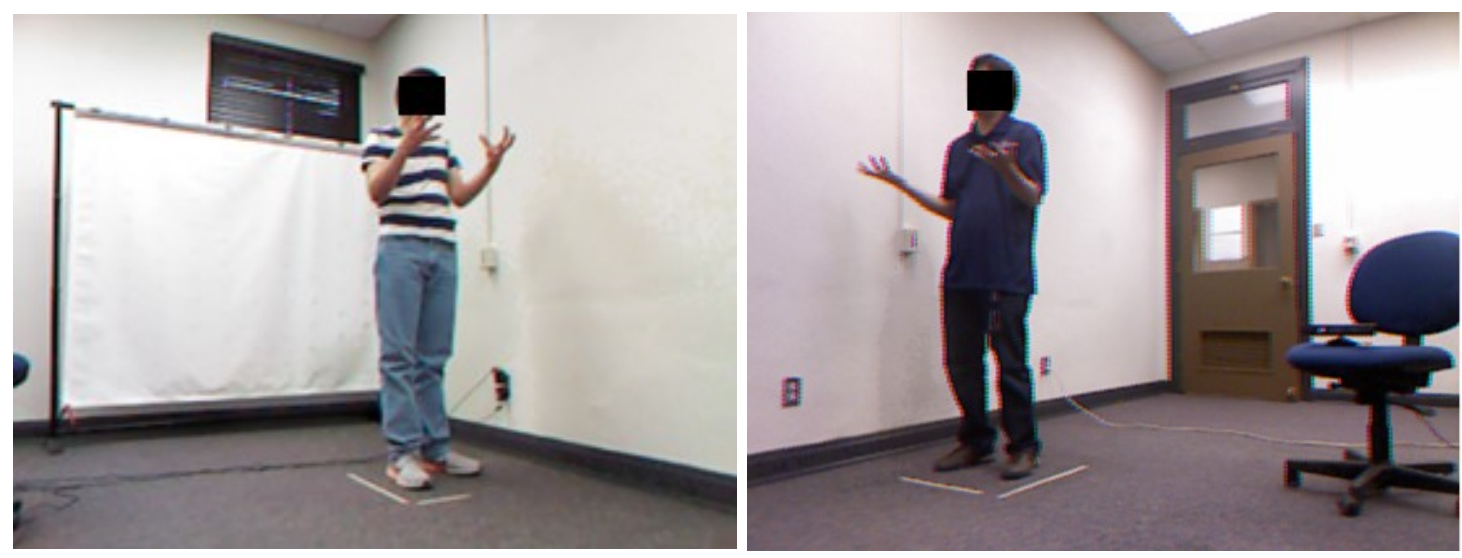

Figure 4. Two participants conversing. The introverted subject (left) uses the same Arms Out gesture as the extravert (right) but his arms are rotated inwards closer to his body.

A conversation topic was given to the pair so that they would begin a discussion, then after five minutes an experimenter would interrupt the conversation and ask them change to a different topic. The topics chosen were made to encourage discussion between subjects: "What favorite movie do you have in common?" and "Create a story about an accident occurring within the Chemistry and Computer Science Building."

\subsubsection{Procedure}

From the recordings, each video was hand-annotated by counting the number of gestures. Each gesture was categorized as a gesture type to determine if there were any specific gestures that were used more frequently across personality types. The first category is whether the subject performing the gesture fidgeted frequently during the gesture. For this experiment, fidgeting is defined as small repeated movements made during a gesture. The second category is the gesture type (deictic, beat, or iconic). The third category is the gesture that was performed. My 
colleagues and I developed this list of gestures, presented in Table 1, based on a previous analysis of these videos. We categorized the gestures in terms of physical characteristics rather than by (what would have to be presumed) meaning. Table 2 presents three examples of annotations.

Table 1. List of types of observed gestures.

\begin{tabular}{|l|l|}
\hline $\begin{array}{l}\text { One hand away from } \\
\text { body (front) }\end{array}$ & $\begin{array}{l}\text { Both hands away from } \\
\text { body (front) }\end{array}$ \\
\hline $\begin{array}{l}\text { One hand away from } \\
\text { body (side) }\end{array}$ & $\begin{array}{l}\text { Both hands away from } \\
\text { body (side) }\end{array}$ \\
\hline One hand on chin & Both hands on chin \\
\hline $\begin{array}{l}\text { One hand in front of } \\
\text { face }\end{array}$ & $\begin{array}{l}\text { Both hands in front of } \\
\text { face }\end{array}$ \\
\hline $\begin{array}{l}\text { One hand on top of } \\
\text { head }\end{array}$ & $\begin{array}{l}\text { Both hands on top of } \\
\text { head }\end{array}$ \\
\hline One arm across chest & Both arms across chest \\
\hline One hand on hip & Both hands on hip \\
\hline One hand below hip & Both hands below hip \\
\hline One hand on back & Both hands on back \\
\hline Touching arm & Hands together \\
\hline Shrug & Other \\
\hline
\end{tabular}

Table 2. Annotations of three beat gestures.

\begin{tabular}{|c|c|c|c|c|}
\hline Gest & $\begin{array}{l}\text { Is } \\
\text { fidget? }\end{array}$ & I/D/B? & $\begin{array}{l}\text { One hand } \\
\text { away from } \\
\text { body (front) }\end{array}$ & $\begin{array}{l}\text { Both hands away } \\
\text { from body (front) }\end{array}$ \\
\hline 1 & 0 & beat & & 1 \\
\hline 2 & 0 & beat & & 1 \\
\hline 3 & & beat & 1 & \\
\hline
\end{tabular}

To ensure accuracy of the annotations, three annotators were assigned to rate each of the videos. None of the annotators knew the personality traits of the subjects. All gestures that were performed by a subject were segmented from the original 13 videos so that each gesture could be rated individually. In total, there were 392 segmented gesture videos. The annotation process consisted of playing the video of a gesture, on repeat, until each annotator was finished rating. Annotators were not allowed to say what they rated until all were finished. However, they could 
discuss aspects that were unclear about a gesture like the start and endpoint and description of the annotation categories. Figure 5 shows a layout of the room where the videos were projected.

Subjects had to stand and talk to one another, which means most of the gestures that occurred were related to the arms and hands. It was expected, from both a previous study (Hostetter, 2012) and from viewing the conversations on my own, that extraverts would gesture more frequently than introverts. I did not have a priori expectations about differences between extraverts and introverts with respect to frequency distributions of their kinds of gestures, as this phase of the study was exploratory.

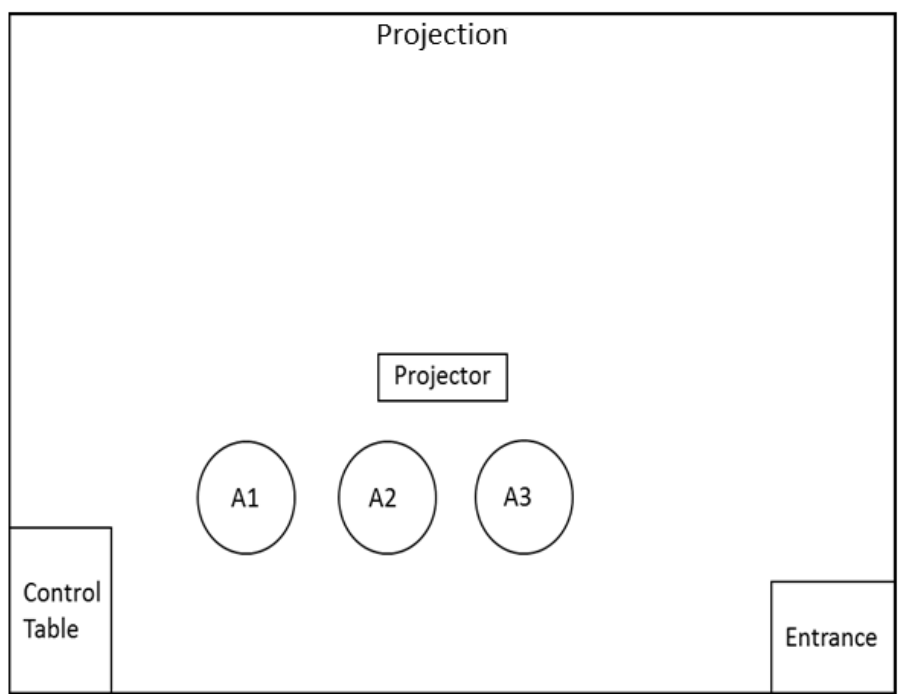

Figure 5. The annotators were in the same room but were only allowed to converse about a gesture after everyone had rated it.

\subsubsection{Equipment}

For recording the participants, two Microsoft Kinects were used to each record one of the subjects in the room. A projector displayed the gestures so that the annotators could rate them without having to each individually own a copy of all the gesture videos. Annotators used their own laptops to record their results in Excel files so that they could not look at each other's ratings. 


\section{Chapter 4: Results}

As each study is related to each other in some capacity, the results section will start with what I found about measuring gesture amplitude. This study gives greater insight into the gesture perception results, and I will conclude by detailing the gesture frequency results. It is important to note that these studies were exploratory in nature, the results give an indication of where future work can be continued. Also each study shares the same corpus, which is the recorded one-to-one conversations, but uses the recordings differently. The agent's gestures were created from these recordings, and the gestures from them were categorized into the $\mathrm{A}, \mathrm{B}$, and $\mathrm{C}$ categories for the measurement and perception studies. The frequency study uses the recordings directly for annotation.

\subsection{Amplitude Measurement Results}

After measuring each of the gestures and calculating the left- and right-arm vectors, I sorted the gestures into three categories according to size: small, medium, and large. Gestures were sorted into categories by thirds of the total amount of gestures (i.e. ten gestures in each category). There is no established way to divide the gestures by the vector sum ranges since this is the first study to do sort by amplitude. Each gesture was measured in inches. The vector length of each gesture can be seen in Appendix A. As a reminder, the A, B, and C gestures were recorded from video conversations and implemented into the agent. The I and E gestures were created by the lab in a previous experiment and were meant to act as introverted (I) and extraverted (E) gestures. The E gestures were just amplified versions of the I gestures. As expected, the smallest gestures were

mostly from the A and B gestures. The medium gestures contained most of the I gestures. The $\mathrm{C}$ and $\mathrm{E}$ gestures composed the majority of the large gestures. Table 3 presents these groupings. 
Table 3. The amplitude vector for each arm was added and the gestures were sorted to create an even distribution across the small, medium, and large categories.

\begin{tabular}{|c|l|}
\hline $\begin{array}{c}\text { Gesture Size by Vector } \\
\text { Sum }\end{array}$ & \multicolumn{1}{|c|}{ Gesture Labels } \\
\hline Small (Max below 14) & $\begin{array}{l}\text { A2, B5, A3, A1, B2, } \\
\text { B4, B6, I4, A4 }\end{array}$ \\
\hline $\begin{array}{c}\text { Medium (Max between } \\
\text { I5, I2, I3, C5, I1, A5, } \\
\text { 15 and 32) }\end{array}$ & $\begin{array}{l}\text { C3, E5, E2, C4, E4 } \\
\text { E1, C2, A6, B1, C1, E6, }\end{array}$ \\
\hline \begin{tabular}{c} 
Large (Sum above 32) \\
\hline
\end{tabular}
\end{tabular}

As indicated in Figure 6, overall the gesture stimuli ranged in amplitude from the A gestures as the smallest, through the B, I, and C gestures, to the E gestures, which were the largest. For reference, the agent's height is 60 inches tall with an arm span of 59 inches when she is projected on the wall. The categorization of these gestures could provide a baseline for gestures that need to be made in the future if they correlate with the perceived naturalness in the next set of results.

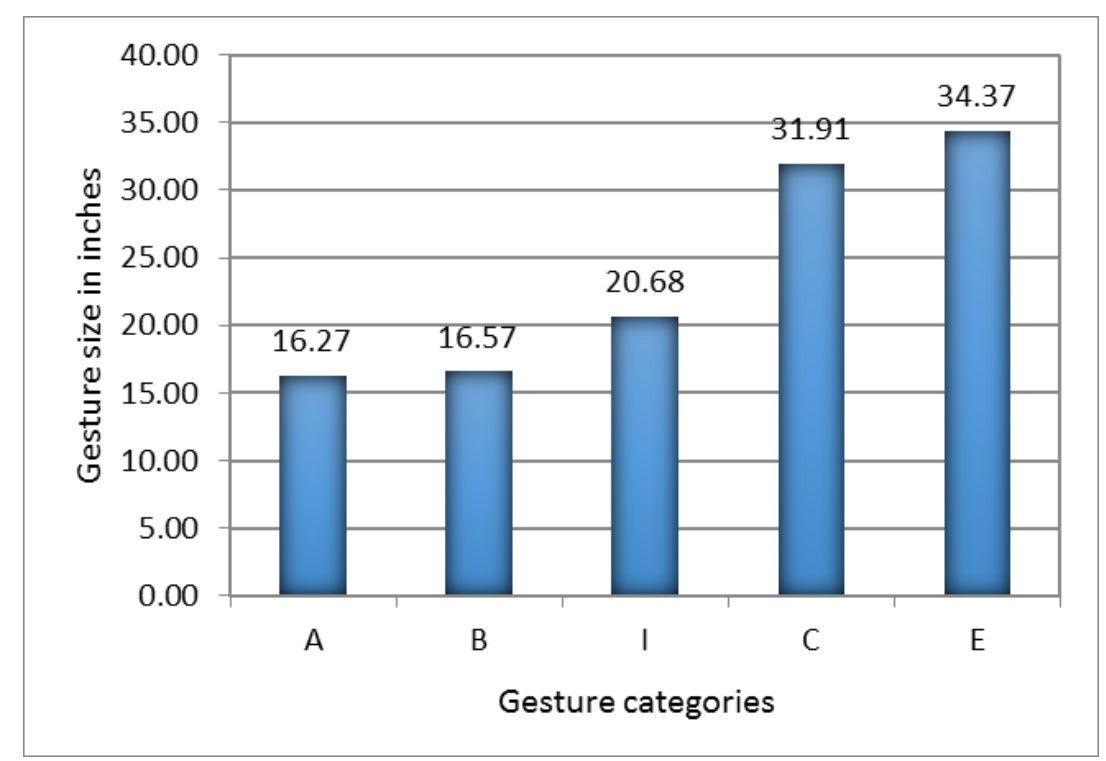

Figure 6. Mean amplitudes (in inches) of the gestures to be used in the perception study. Each gesture amplitude was calculated as the maximum of the the right-arm and left-arm vectors. 


\subsection{Amplitude Perception Results}

I calculated a mean rating for each type of animation by taking the average of the ratings of each gesture across all participants (i.e. average rating of A1) and then calculating the average for each category of gestures (i.e. average of A1 to A6 is mean rating of A animations). This was done for both introverts and extraverts. Unsurprisingly, the subjects' judgments of amplitude tracked the actual amplitudes of the gestures. The mean rating for the A gestures, which had smallest amplitude, was 2.85, that for the E gestures, which had the largest amplitude, was 4.21. This difference was significant (paired t-test, 2 tails, $\mathrm{p}<0.0001$ ). The correlation between judgments of introverts and extraverts was 0.95 , which suggests that introverts and extraverts perceive gesture amplitude similarly. Figure 7 shows each gesture category's mean rating of amplitude; a rating of 4 represents the most natural amplitude (the middle of the rating scale given to the participants).

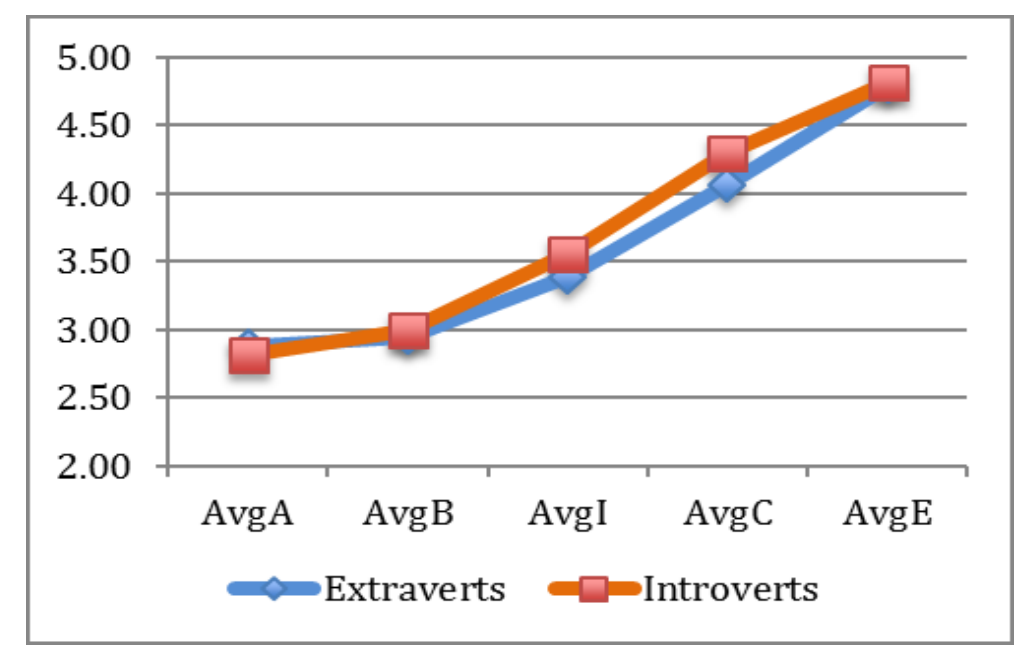

Figure 7. The rating of amplitude overall for each set of animations (A to $\mathrm{C}$ increasing in amplitude, I and E previously used animations). A score of 4 represents the amplitude judged most natural

The $\mathrm{C}$ and $\mathrm{E}$ gestures are relatively close to 4, which means they are viewed as the most natural. Figure 8 shows the subjects' combined mean amplitude ratings as a function of the gestures' actual amplitude, as measured by the maximum of the right- or left-hand gesture vector. 
The resulting plot is almost linear and seems to plateau at the size of the $\mathrm{C}$ and $\mathrm{E}$ gestures. As speculation, these categories may represent an amplitude limit, and anything larger could mean that naturalness will decrease.

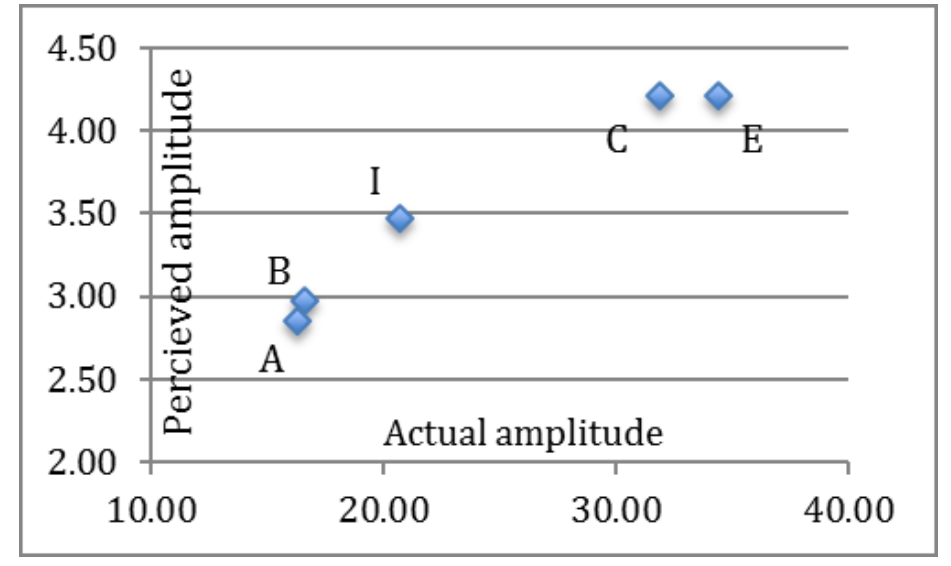

Figure 8. Perceived amplitude vs. actual amplitude. A score of 4 represents the amplitude judged most natural. The actual amplitude represents the maximum of the the right-arm and left-arm vectors.

The maximum vector between the left and right arm, median for extraverted naturalness, and median for introverted naturalness were calculated and are presented in Table 4. The medians suggest that gestures with a left- or right-arm vector from 26 to 35 inches were seen as the most natural across both personality types. For clarity, Figure 9 presents this relationship as a chart.

Table 4. Median for each amplitude category and its relation to the naturalness perception study ratings (higher $=$ more natural).

\begin{tabular}{|c|c|c|c|}
\hline & $\begin{array}{c}\text { Maximum } \\
\text { L-R Vector }\end{array}$ & $\begin{array}{c}\text { Extravert } \\
\text { Naturalness }\end{array}$ & $\begin{array}{c}\text { Introvert } \\
\text { Naturalness }\end{array}$ \\
\hline Small & 6.73 & 2.50 & 2.54 \\
\hline Medium & 26.31 & 3.13 & 3.09 \\
\hline Large & 34.83 & 3.13 & 3.14 \\
\hline
\end{tabular}




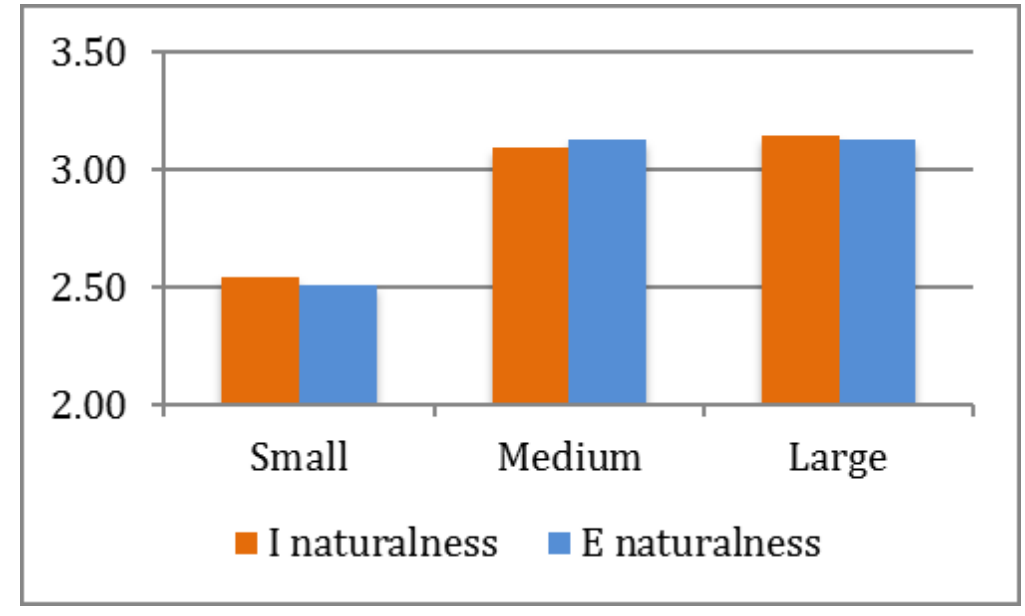

Figure 9. Median for each amplitude category and its relation to the naturalness perception study ratings (higher $=$ more natural).

\subsection{GeSture Frequency Results}

I now turn to the research question about the relative frequencies of gestures across personality types. I performed a Fliess's Kappa test on five annotated videos to ensure validity of agreement. All Kappas were greater than 0.8, which suggests that the annotators had excellent agreement.

The data suggest that extraverts do gesture more frequently than introverts. The mean gesture frequencies, in gestures per minute, were 4.11 (extravert) and 2.84 (introvert). The standard deviations were high for both groups: 2.06 (extravert) and 1.35 (introvert). However, even with the large main effect, the small sample size and high variance led to a t-test result that was only suggestive $(p=0.075)$. The main effect is consistent with the findings reported in Hostetter's study (Hostetter, 2012), but in this case for conversation that could be seen as more natural.

I also assessed frequencies for beat and non-beat ("representational") frequencies. For nonbeat gestures, corresponding to those reported in Hostetter's study (Hostetter, 2012), the mean gesture frequencies, in gestures per minute, were 1.74 (extravert) and 0.94 (introvert). The standard deviations were again relatively high: 1.22 (extravert) and 0.76 (introvert). The results of a t-test were likewise only suggestive $(p=0.091)$. These results are consistent with those found in Hostetter's study (Hostetter, 2012). For the beat gestures, the differences between extraverts and 
introverts did not appear to be as pronounced as for the non-beat gestures. For the beat gestures, the mean gesture frequencies, in gestures per minute, were 2.61 (extravert) and 2.07 (introvert). The standard deviations were 1.44 (extravert) and 0.67 (introvert). The results of a t-test were correspondingly inconclusive $(\mathrm{p}=0.14)$.

Interestingly, extraverts displayed a greater range of gesture frequencies than introverts. Indeed, some of the extraverts gestured at the same rate as some of the introverts. Overall gesture frequencies, in gestures per minute, ranged from 0.68 to 6.87 (extravert) and from 1.90 to 5.53 (introvert). The mean frequency, minimum, and maximum frequency are presented in Table 5.

Table 5. The minimum and maximum gestures per minute for each personality type, along with the average.

\begin{tabular}{|c|c|c|}
\hline Gestures/Minute & Extraverts & Introverts \\
\hline Mean & 4.11 & 2.84 \\
\hline Minimum & 0.68 & 1.90 \\
\hline Maximum & 6.87 & 5.53 \\
\hline
\end{tabular}

As to differences between introverts and extraverts with respect to types of gestures performed, I compared the relative occurrences of iconic, deictic, and beat gestures across the extravert and introvert subject groups. A Chi-Square analysis suggests that the distributions are not dissimilar $(\mathrm{p}=0.94)$.

Although the distributions are not dissimilar, I wanted to see if there may be any trends between gesture frequencies of all the gestures. I calculated relative gesture frequencies for the 22 categories of specific gesture listed in Table 1. Figure 10 presents the relative frequencies of the 19 categories of gesture for which at least one gesture was observed in the corpus. (I found no instances of both-hands-on-chin or both-hands-on-top of head.) The results in Figure 10 suggest that extraverts tend with greater frequency to gesture with both hands to the side of the body, and introverts tend with greater frequency to gesture with one hand in front of face, with one hand on 
top of the head, and with bringing the hands together in front of the body. Additionally, it appears that extraverts tended to use a wider range of gestures than did introverts, in that the distribution of gestures for extraverts was less uniform than that that for introverts: the average frequency across all gesture types was $4.55 \%$. This means that on average each gesture type was used about $4.55 \%$ across all the conversations for both personality types. Between personality types though, the variance of average gesture frequencies for extraverts was $0.46 \%$ and for introverts was $0.38 \%$. These frequencies lead to believe that extraverts use a wider range of gestures, but a larger corpus would be required to see if this idea holds.

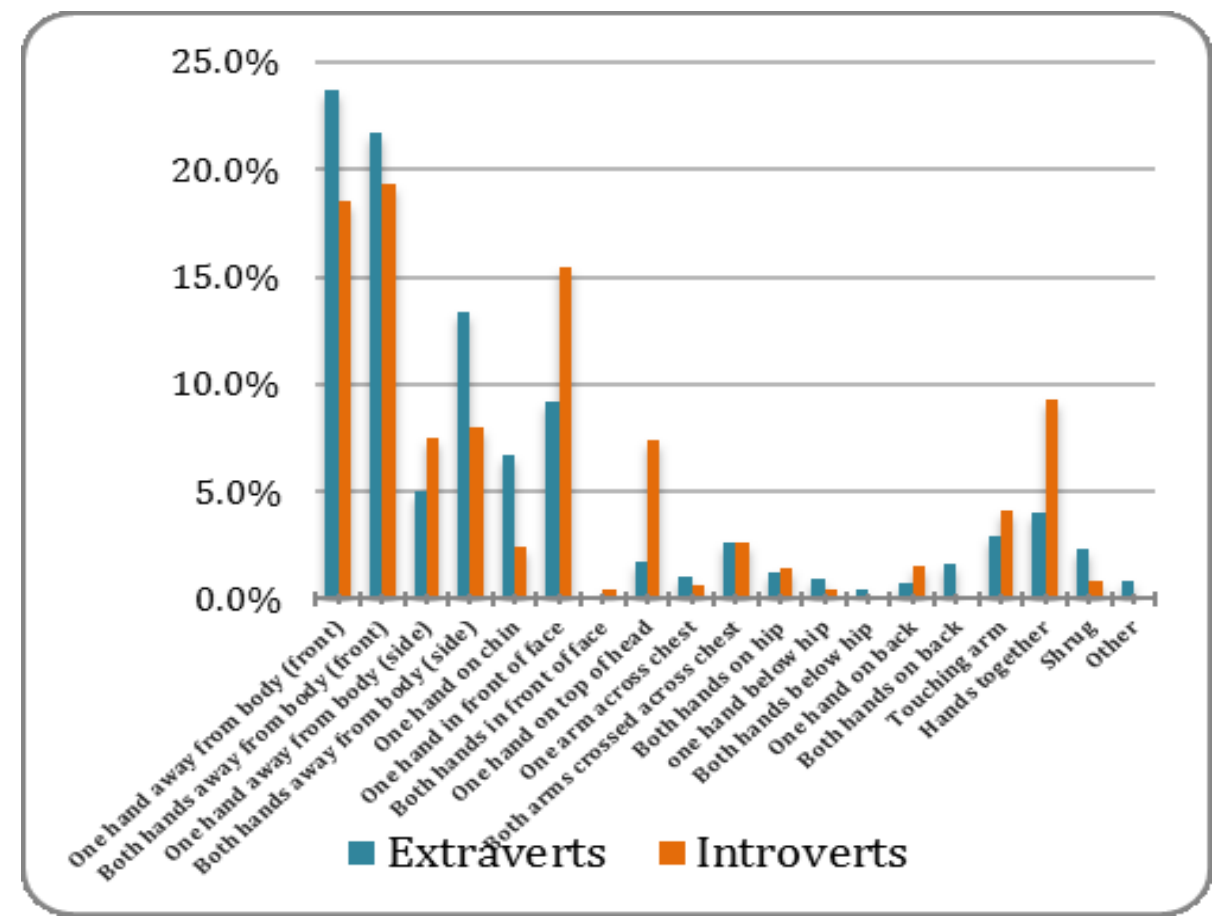

Figure 10. Average distribution for observed gestures. 


\section{Chapter 5: Discussion}

In this chapter I review the three main questions of this thesis in light of the results reported in Chapter 4. This thesis is exploratory in nature and has presented the first known account of the relationship between gesture and personality that reports the precise amplitudes of the gestural stimuli. Research into gestural amplitude for virtual agents is still in its infancy, however. The results reported here can serve as a stepping-stone in understanding how to design agent gestures.

\subsection{Gesture AMPLitude Discussion}

In this section I will discuss the first two main research questions:

1. Do people perceive an agent's gesture as more or less natural as a function of (a) the person's personality trait of extraversion vs. introversion and (b) the amplitude of the agent's gestures?

2. Do the perceived sizes of the gestures correspond to their actual sizes?

The results suggest that extraverts and introverts tend to see gesture amplitudes similarly in terms of perceived naturalness. That is, people do not appear to perceive an agent's gesture as more or less natural as a function of the person's personality trait of extraversion vs. introversion. With respect to some classes of gesture amplitude, both extraverts and introverts seem to be able to distinguish differences in the naturalness of gestures that differ by amplitude. In other words, people do appear to perceive an agent's gesture as more or less natural as a function of the amplitude of the agent's gestures.

This is helpful for developers of ECAs because the size of gestures need not be changed as a function of personality types of users. However, a limitation of this study is that these measurements for the vectors may be relative to the size of our agent. The agent is about 60 inches tall with an arm span of 59 inches. These results may not be valid for an agent on a smaller screen. But agents of a similar size should benefit from the amplitude measurements developed in this thesis. 
The gestures used by the agent had no finger movement and in a very few instances had slight clipping. Even though participants were told to ignore these limitations, these qualities still may have affected the ratings. As a reminder, the perception study did not consider the context of the gesture. In a conversational setting the context of the gesture as well as the size could change the perceived naturalness of the gesture.

The gestures used by the agent in this study correlated with the perceived levels of naturalness by the participants. This supports the validity of the gesture-size rubric developed for the agent. Any gestures that need to be created in the future of the agent, or similarly sized agents can follow the rubric in this thesis. From the rubric, it is safe to say that gestures from the C, E, and some of the I category are optimal for creating the most natural gestures. The movements in the A and B category may have been rated low because of their, in some cases, almost staticlooking movement. In most cases of the A gestures, the agent was barely moving her arms and hands to the sides of her body. These movements are comparable to weight-shift animations. The B gestures are similar, but a few of them contain slightly larger displacements. There was an outlier gesture, A6, whose measurements can be seen in Appendix A. The left hand vector is 0 while the right hand vector is large because this gesture was captured from a person who was leaning against a wall. Regardless, these gestures were captured from live conversations of mixed-personality pairs. The participants had never met each other before their conversations, which could explain the stilted movement.

\subsection{GESTURE FREQUENCY DISCUSSION}

In this section I discuss the third set of research questions:

3. Which personality type gestures more frequently, and are there any gestures that are more frequently used by each one?

With respect to gesture frequencies, the results suggest that extraverts gesture with greater frequency than introverts, although this trend appears to be more pronounced for iconic and deictic gestures than for beat gestures. The variance in gesture frequency was much higher for extraverts 
than for introverts, so much so that some of the extraverts gestured less frequently than any of the introverts.

Conversants' types of gestures (iconic, deictic, and beat) do not appear to depend on the personality type, although extraverts have a greater range of variance in performing their gestures. The extraverts have a gesture frequency range of 0.68 to 6.87 gestures per minute, while the introverts are 1.9 to 5.53 gestures per minute. The introverts' range is encapsulated into the extraverts' range. This means that an extravert can perform a wide variety of gestures yet appear introverted due to the variance. After annotation, the annotators were surprised when they had found out a person was introverted or extraverted because of how frequently they had gestured. There are apparent differences in the relative frequencies of some specific gestures as seen in Figure 10, such as both hands to the side of the body (higher frequency for extraverts) and one hand on the chin, one hand on top of the head, and bringing the hands together in front of the body (higher frequency for introverts). Why each personality type uses these specific gestures is not known. It is possible that other personality traits may affect frequency, but this would need to be tested in future work.

As for whether fidgeting occurred more between introverts and extraverts, almost no subjects fidgeted. I had defined fidgeting as small repeated movements made during a gesture. This is not to be confused with beat gestures, which conversants use to emphasize words by pausing the rhythm of speech. I had included fidgeting as part of the features because I thought it would be a characteristic of introversion, which would be important to integrate for an introverted agent. From this sample of conversations, it does not look like fidgeting is an important quality for either personality type.

All of this should be helpful for developers of ECAs because an agent can perform gestures with different frequencies and distributions and could still be perceived as extraverted. Developers can coordinate their agents' gesture by using the desired gesture per minute frequencies that was developed in Table 5. An interesting note is that usually the frequency changes often within conversation, and if an agent keeps a constant frequency it could be unnatural. 
The validity of my study is limited by the small number of conversations that were recorded and annotated. A few of the videos that had been recorded had become corrupted as well, which left just the 13 to use. The results could differ if a larger sample size were taken. The culture and gender may influence frequency as well. This study was held in a mostly Hispanic community, with almost all of the participants coming from this community. The difference between introvert and extravert frequency could vary by what culture they grew up in; coming from the same culture where many people here gesture with their hands to bring points across.

\subsection{FUTURE WORK}

This thesis is another step in discovering how much of an effect gesture amplitude and frequency can have on human-agent interaction. For other researchers looking to continue from the work presented here, I recommend looking at several avenues to ensure the validity of the results. The first would be testing the scalability of the amplitude measurement and perception studies. Agents can range visually not just in the way they are presented to users (i.e., projection, virtual reality, computer screen) but also by physical features. The perceived naturalness could change if the agent is short and stocky as opposed to tall and lanky or anywhere in-between. Discovering a scalability ratio between the agent's height and the size of their gesture would be the first stepping off point to accomplishing natural gestures for any agent. Another interesting subject is testing the results here in a conversational setting with a similar agent. For the amplitude study participants judged only the agent's gesture size without the agent having speech. I believe that adding dialogue would only help to contribute to the level of perceived extraversion from the agent, but no experiments have been tested in a conversational setting involving the user directly. The storytelling agents from Hu's work (2015) and Clausen-Bruun's videos (2013) did not directly interact with the user. The user acted as a third party listening to the agent. Another issue that could arise would be that, in a conversational setting, gestures should flow into one another. The agent was reset to her starting position each before each gesture was played. Having the gestures "connect" could lead to different results in terms of perception of the size. 
As mentioned before, the frequency (and possibly the amplitude) results may vary from culture, gender, and level of rapport between the people we had recorded. In all cases, all of the participants met each other for the first time. Not only that, but only 13 videos were usable so a larger corpus may alter the results significantly. It is my suggestion that a larger, more diverse personality trait and population sample should be annotated. Another idea is to test what was found here regarding the gestures per minute ranges for extraverts and introverts with an agent. The agent could alternate its gesture rate by that of extravert data. It may facilitate the level of perceived extraversion personality, or possibly harm it depending on how frequent the agent adjusts its rate.

Another avenue to look into would be how other personality dimensions of the Big Five affect amplitude and frequency. Agreeableness, openness to experience, conscientiousness, and neuroticism have seen some interest in regard to frequency (Hostetter, 2012) but not so much with amplitude. 


\section{Chapter 6: Conclusion}

Research into virtual agents and how we can best design them to develop rapport is composed of many factors. This thesis is aimed at improving one of these factors: non-verbal gestures. Gestures represent the personality of an agent and in turn that personality affects how user's will develop rapport with the agent (Gratch, 2007). Gesture amplitude and frequency are important parts of creating humanlike gestures as well as display the personality of an agent (Neff et al., 2008; Neff et al., 2010). Previous literature had not made clear what the difference between an extraverted and introverted gesture is. This thesis has created empirical guidelines to follow when designing an agent's gestures to display an extraverted personality. To appear natural, agents can use gestures within the given amplitude size vectors and can use differing degrees of frequency to appear extraverted.

The ideal size for natural gesture is 26 to 34 inches for the arms. These measurements apply to the agent used in this thesis, but could be scalable. Regardless of the personality type of the user interacting with the agent, subjects will likely judge the naturalness of the agent's gesture amplitude the same. It is not completely clear whether introverts and extraverts use specific gestures or gesture types more often than one another. There are some indications that extraverts can appear introverted through their frequency.

These three studies on gesture frequency and amplitude are exploratory. The results suggest that frequency and amplitude do contribute to the personality and naturalness of the agent. The next step would be to test these findings in a conversational setting between the agent and user. It would be interesting to see how users would develop rapport with an agent that had the same dialog, but different gesture amplitude and frequency in a conversational setting.

Another area to consider would be if a constant frequency or size would be noticed, or appear unnatural. If this is not the case, then the amplitude and frequency for all agents could be standardized even further. I hope this thesis inspires others to look deeper into how we as 
researchers design our agents. Even the smallest gesture could influence the agent's personality and naturalness. 


\section{References}

Alexei V. Ivanov, et al. "Recognition of personality traits from human spoken conversations." Interspeech (2011): 1549-1552.

Argyle, M.: “Bodily communication.” Taylor \& Francis (1988)

Bergmann, K., Kopp, S., \& Eyssel, F. (2010, September). "Individualized gesturing outperforms average gesturing-evaluating gesture production in virtual humans." In International Conference on Intelligent Virtual Agents, 104-117.

Bickmore, Timothy, and Justine Cassell. "Relational agents: a model and implementation of building user trust." In Proceedings of the SIGCHI conference on human factors in computing systems, pp. 396-403. ACM, 2001

Bond, P. (2011). "Video game sales on winning streak, study projects." Reuters. Retrieved (2011): 03-12.

Botwin, Michael D., David M. Buss, and Todd K. Shackelford. "Personality and mate preferences: Five factors in mate selection and marital satisfaction." Journal of Personality 65.1 (1997): 107-136.

Brixey, J. E. "Virtual rapport with extraverted agents." University of Texas at El Paso CS Department Master's Thesis (2015).

Cafaro, Angelo, Hannes Högni Vilhjálmsson, Timothy Bickmore, Dirk Heylen, Kamilla Rún Jóhannsdóttir, and Gunnar Steinn Valgarðsson. "First impressions: Users' judgments of virtual agents' personality and interpersonal attitude in first encounters." In Intelligent Virtual Agents, pp. 67-80. Springer Berlin Heidelberg, 2012

Cassell, Justine. “Embodied conversational agents.” MIT press, 2000.

Cerekovic, A., Aran, O., \& Gatica-Perez, D. (2014, September). "How do you like your virtual agent?: Human-agent interaction experience through nonverbal features and personality traits." In International Workshop on Human Behavior Understanding, 1-15.

Clausen-Bruun, Mette, Tobias Ek, and Magnus Haake. "Size certainly matters-at least if you are a gesticulating digital character: The impact of gesture amplitude on addressees' information uptake." Intelligent Virtual Agents. Vol. 446. 2013.

Digman, John M. "Personality structure: Emergence of the five-factor model." Annual review of psychology 41.1 (1990): 417-440.

Eysenck, Sybil BG, Hans J. Eysenck, and Paul Barrett. "A revised version of the psychoticism scale." Personality and individual differences 6, no. 1 (1985): 21-29

Gratch, Jonathan, Ning Wang, Anna Okhmatovskaia, Francois Lamothe, Mathieu Morales, Rick J. van der Werf, and Louis-Philippe Morency. "Can virtual humans be more engaging than real ones?."In Human-Computer Interaction. HCI Intelligent Multimodal Interaction Environments, pp. 286-297. Springer Berlin Heidelberg, 2007.

Gratch, Jonathan, et al. "Creating rapport with virtual agents." Intelligent Virtual Agents (2007): 125-138. 
Gris, Ivan, Diego A. Rivera, and David Novick. "Animation guidelines for believable embodied conversational agent gestures." International Conference on Virtual, Augmented and Mixed Reality. Springer International Publishing, 2015.

Hostetter, Autumn B., and Andrea L. Potthoff. "Effects of personality and social situation on representational gesture production." Gesture 12.1 (2012): 62-83.

Hu, C., Walker, M. A., Neff, M., \& Fox Tree, J. E. F. (2015, August). "Storytelling agents with personality and adaptivity." In International Conference on Intelligent Virtual Agents, 181-193.

Jung, C.: "Psychological types." In the collected works of C. G. Jung. Volume 6., Princeton, NJ, Princeton University Press (1971, originally 1921)

Kendon, A. (1980). "Gesticulation and speech: Two aspects of the process of utterance." In M.R. Key (Ed.) The Relationship of Verbal and Nonverbal communication. The Hague, NL: Mouton Publishers.

Kendon, A. (1986). "Some reasons for studying gesture." Semiotica 62, 3-28.

Kendon, A. (1994). "Do gestures communicate?: A review." Research on Language and Social Interaction, 27, 175-200.

Kipp, Michael; Kipp, Kerstin H.; Ndiaye, Alassane; Patrick (2006), "Evaluating the tangible interface and virtual characters in the interactive COHIBIT exhibit", Proceedings of the International Conference on Intelligent Virtual Agents, 434-444.

McNeill, D. (1985). "So you think gestures are nonverbal?" Psychological Review, 3, 350371.

Mehrabian, A. and Ferris, S.R. (1967). "Inference of attitude from non-verbal communication in two channels." The Journal of Counselling Psychology, 31, 248-252.

Myers, I.B., Myers, P.B.: "Gifts differing." Consulting Psychologists Press (1980)

Neff, M., Kipp, M., Albrecht, I., and Seidel, H. P. (2008). "Gesture modeling and animation based on a probabilistic re-creation of speaker style." ACM Transactions on Graphics, $27(1), 5$.

Neff. M., Wang, Y., Abbot, R. and Walker, M. (2010). "Evaluating the effect of gesture and language on personality perception in conversational agents." In J. Allbeck et al. (Eds.): Proceedings of the International Conference on Intelligent Virtual Agents, 222-235

Niedenthal, Paula M., et al. (2010). "The Simulation of Smiles (SIMS) model: Embodied simulation and the meaning of facial expression." Behavioral and Brain Sciences 33.06. 417-433.

Novick, David, and Gris, I. (2014) "Building rapport between human and ECA: A pilot study." International Conference on Human-Computer Interaction, 472-480.

Novick, David, et al. "The UTEP AGENT System." Proceedings of the 2015 International Conference on Multimodal Interaction. ACM, 2015.

"Jung Word Pair Test.” Retrieved from http://similarminds.com/jung_word_pair.html 
Robert R. McCrae and Paul T. Costa Jr. "Personality trait structure as a human universal," American Psychologist 52, no. 5 (1997): 509.

Swartout, William, et al. "Ada and Grace: Toward realistic and engaging virtual museum guides." International Conference on Intelligent Virtual Agents. Springer Berlin Heidelberg, 2010. 


\section{Appendix}

Appendix A

The vector for each arm was measured and the maximum between the left and right arm vectors was chosen as the representative for the size of the gesture.

\begin{tabular}{|l|l|l|l|}
\hline & Right Hand Vector & Left Hand Vector & Greatest Amplitude Vector \\
\hline A1 & 6.70 & 3 & 6.70 \\
\hline A2 & 4.12 & 3 & 4.12 \\
\hline A3 & 5.74 & 5.74 & 5.74 \\
\hline A4 & 13.67 & 3.16 & 13.67 \\
\hline A5 & 25.67 & 26.34 & 26.34 \\
\hline A6 & 41.03 & 0 & 41.03 \\
\hline
\end{tabular}

\begin{tabular}{|l|l|l|l|}
\hline & Right Hand Vector & Left Hand Vector & Greatest Amplitude Vector \\
\hline B1 & 34.79 & 12.88 & 34.79 \\
\hline B2 & 6.73 & 4.53 & 6.73 \\
\hline B3 & 1.00 & 32.70 & 32.70 \\
\hline B4 & 8.92 & 7.28 & 8.92 \\
\hline B5 & 4.92 & 4.00 & 4.92 \\
\hline B6 & 11.36 & 8.00 & 11.36 \\
\hline
\end{tabular}




\begin{tabular}{|l|l|l|l|}
\hline & Right Hand Vector & Left Hand Vector & Greatest Amplitude Vector \\
\hline C1 & 34.83 & 21.93 & 34.83 \\
\hline C2 & 39.13 & 25.29 & 39.13 \\
\hline C3 & 27.21 & 23.22 & 27.21 \\
\hline C4 & 6.40 & 31.78 & 31.78 \\
\hline C5 & 23.18 & 25.02 & 25.02 \\
\hline C6 & 33.50 & 20.83 & 33.50 \\
\hline
\end{tabular}

\begin{tabular}{|l|l|l|l|}
\hline & Right Hand Vector & Left Hand Vector & Greatest Amplitude Vector \\
\hline I1 & 25.42 & 4.03 & 25.42 \\
\hline I2 & 16.58 & 18.97 & 18.97 \\
\hline I3 & 16.36 & 19.29 & 19.29 \\
\hline I4 & 10.11 & 11.92 & 11.92 \\
\hline I5 & 16.07 & 13.67 & 16.07 \\
\hline I6 & 32.41 & 5.00 & 32.41 \\
\hline
\end{tabular}

\begin{tabular}{|l|l|l|l|}
\hline & Right Hand Vector & Left Hand Vector & Greatest Amplitude Vector \\
\hline E1 & 37.01 & 4.12 & 37.01 \\
\hline E2 & 31.19 & 31.10 & 31.19 \\
\hline E3 & 39.48 & 38.27 & 39.48 \\
\hline E4 & 21.37 & 31.95 & 31.95 \\
\hline E5 & 30.48 & 28.72 & 30.48 \\
\hline E6 & 36.12 & 6.08 & 36.12 \\
\hline
\end{tabular}




\section{Vita}

Alex Michael Rayon was born in San Antonio, Texas and earned his Masters from the University of Texas at El Paso. He has been building embodied conversational agents at UTEP's Interactive Systems Group as part of the Advanced Agent Engagement Team. He developed the agent and virtual environment for Survival on Jungle Island, a research project on rapport that won best demonstration at ICMI 2015. Alex co-founded GameBuilders!, a student organization at UTEP where members learn about the work behind creating immersive environments and ECAs to create unique interactive games. Alex is also the co-founder of Inmerssion where he creates virtual reality experiences utilizing what he has learned from his research. He likes to create believable virtual characters for each project by fine tuning their movement, dialogue, and personas.

Contact Information: 2029 Paseo Del Prado Dr.

El Paso, TX, 79936

This thesis was typed by Alex Michael Rayon. 